Putative orthologous relations between two genomes are defined as gene couples satisfying the bi-directional best hit (BBH) criterion or a blastP alignment threshold, a minimum of $\mathbf{3 5 \%}$ sequence identity on $\mathbf{8 0 \%}$ of the length of the smallest protein. These relations are subsequently used to search for conserved gene clusters, e.g. synteny groups (syntons) among several bacterial genomes. All possible kinds of chromosomal rearrangements are allowed (inversion, insertion/deletion). A gap parameter, representing the maximum number of consecutive genes which are not involved in a synteny group, is set to five genes.

limit set: $50 \%$ of the genes in synton. In red: PKGDB in black refseq

$$
\text { QUERY }
$$

\section{CDS in}

Syntons $\quad$ BBH

$\underline{\mathrm{Nb}} \quad \underline{\%} \quad \underline{\mathrm{Nb}} \quad \underline{\%}$

$\begin{array}{llll}3415 & 80.41 & 3398 & 80.01\end{array}$

$\begin{array}{lllll}3170 & 74.64 & 3297 & 77.63\end{array}$

$\begin{array}{lllll}3135 & 73.82 & 3237 & 76.22\end{array}$

$\begin{array}{llll}3106 & 73.13 & 3153 & 74.24\end{array}$

$\begin{array}{llll}3100 & 72.99 & 3177 & 74.81\end{array}$

$\begin{array}{llll}3054 & 71.91 & 3126 & 73.6\end{array}$

$\begin{array}{llll}3037 & 71.51 & 3102 & 73.04\end{array}$

$\begin{array}{lllll}3025 & 71.23 & 3098 & 72.95\end{array}$

$\begin{array}{llll}3024 & 71.2 & 3074 & 72.38\end{array}$

$\begin{array}{lllll}3018 & 71.06 & 3072 & 72.33\end{array}$

$\begin{array}{llll}3017 & 71.04 & 3088 & 72.71\end{array}$

$\begin{array}{lllll}3014 & 70.97 & 3086 & 72.66\end{array}$

$\begin{array}{lllll}3012 & 70.92 & 3084 & 72.62\end{array}$

$\begin{array}{lllll}3010 & 70.87 & 3047 & 71.74\end{array}$

$\begin{array}{llll}3004 & 70.73 & 3076 & 72.43\end{array}$

$\begin{array}{lllll}3002 & 70.69 & 3056 & 71.96\end{array}$

$\begin{array}{llll}3000 & 70.64 & 3056 & 71.96\end{array}$

$\begin{array}{lllll}2999 & 70.61 & 3060 & 72.05\end{array}$

$\begin{array}{llll}2997 & 70.57 & 3043 & 71.65\end{array}$

$\begin{array}{lllll}2996 & 70.54 & 3044 & 71.67\end{array}$

$\begin{array}{lllll}2988 & 70.36 & 3007 & 70.8\end{array}$

$\begin{array}{lllll}2987 & 70.33 & 3035 & 71.46\end{array}$

$\begin{array}{lllll}2978 & 70.12 & 3020 & 71.11\end{array}$

$\begin{array}{llll}2968 & 69.88 & 3041 & 71.6\end{array}$

$\begin{array}{llll}2964 & 69.79 & 3012 & 70.92\end{array}$ $\begin{array}{llll}2957 & 69.63 & 3023 & 71.18\end{array}$ $\begin{array}{lllll}2954 & 69.55 & 3003 & 70.71\end{array}$ $\begin{array}{llll}2948 & 69.41 & 2979 & 70.14\end{array}$ $\begin{array}{lllll}2948 & 69.41 & 3004 & 70.73\end{array}$

\section{SYNTONS}

Synton size (gene

Synton $\mathrm{Nb}$

$\mathrm{Nb})$

BBH

CDS in

Syntons

$\underline{\text { Min }} \quad \underline{\operatorname{Avg}} \underline{\mathrm{Max}} \quad \underline{\mathrm{Nb}} \quad \underline{\%} \quad \underline{\mathrm{Nb}} \quad \underline{\%}$

$\begin{array}{llllllll}310 & 1 & 12.25 & 198 & 3398 & 74.32 & 3432 & 75.07\end{array}$

$\begin{array}{llllllll}437 & 1 & 8.37 & 95 & 3297 & 60.77 & 3257 & 60.04\end{array}$

$\begin{array}{llllllll}372 & 1 & 9.38 & 115 & 3237 & 67.78 & 3179 & 66.56\end{array}$

$\begin{array}{llllllll}314 & 1 & 10.97 & 138 & 3153 & 63.31 & 3151 & 63.27\end{array}$

$\begin{array}{llllllll}581 & 1 & 6.54 & 69 & 3177 & 49.53 & 3453 & 53.84\end{array}$

$\begin{array}{llllllll}425 & 1 & 8.24 & 109 & 3126 & 53.87 & 3179 & 54.78\end{array}$

$\begin{array}{llllllll}370 & 1 & 9.22 & 109 & 3102 & 59.31 & 3133 & 59.9\end{array}$

$\begin{array}{llllllll}383 & 1 & 8.95 & 110 & 3098 & 58.32 & 3155 & 59.39\end{array}$

$\begin{array}{llllllll}362 & 1 & 9.3 & 114 & 3074 & 65.46 & 3058 & 65.12\end{array}$

370

330

382

385

364

335

340

350

340

370

338

305

352

340

415

310

274

337

361

321
VS

$\underline{\text { CDS Nb }}$ Replicon Name




QUERY

\section{CDS in}

Syntons

$\mathrm{Nb} \quad \% \quad \mathrm{Nb} \quad \%$

$\begin{array}{llll}2947 & 69.39 & 2990 & 70.4\end{array}$

$\begin{array}{lllll}2940 & 69.23 & 2987 & 70.33\end{array}$

$\begin{array}{llll}2938 & 69.18 & 2985 & 70.28\end{array}$

$\begin{array}{llll}2937 & 69.15 & 3020 & 71.11\end{array}$

$\begin{array}{llll}2935 & 69.11 & 2971 & 69.96\end{array}$ $\begin{array}{lllll}2934 & 69.08 & 3004 & 70.73\end{array}$

$\begin{array}{lllll}2919 & 68.73 & 2966 & 69.84\end{array}$

$\begin{array}{lllll}2918 & 68.71 & 3006 & 70.78\end{array}$

$\begin{array}{llll}2916 & 68.66 & 2954 & 69.55\end{array}$

$\begin{array}{llll}2916 & 68.66 & 2944 & 69.32\end{array}$ $\begin{array}{lllll}2910 & 68.52 & 2974 & 70.03\end{array}$

$\begin{array}{llll}2899 & 68.26 & 2943 & 69.3\end{array}$ $\begin{array}{llll}2895 & 68.17 & 2971 & 69.96\end{array}$

$\begin{array}{llll}2890 & 68.05 & 2933 & 69.06\end{array}$

$\begin{array}{llll}2885 & 67.93 & 2923 & 68.83\end{array}$

$\begin{array}{llll}2883 & 67.88 & 2931 & 69.01\end{array}$

$\begin{array}{llll}2877 & 67.74 & 2919 & 68.73\end{array}$

$2873 \quad 67.65 \quad 2897 \quad 68.21$

$\begin{array}{llll}2872 & 67.62 & 2899 & 68.26\end{array}$ $\begin{array}{llll}2869 & 67.55 & 2952 & 69.51\end{array}$

$\begin{array}{llll}2867 & 67.51 & 2901 & 68.31\end{array}$

$2863 \quad 67.41 \quad 2910 \quad 68.52$

$\begin{array}{llll}2861 & 67.37 & 2901 & 68.31\end{array}$ $\begin{array}{llll}2860 & 67.34 & 2896 & 68.19\end{array}$

$\begin{array}{llll}2859 & 67.32 & 2908 & 68.47\end{array}$

$\begin{array}{lllll}2855 & 67.22 & 2883 & 67.88\end{array}$ $\begin{array}{lllll}2853 & 67.18 & 2929 & 68.97\end{array}$

\section{SYNTONS}

\section{Synton size (gene}

$\mathrm{Nb})$

$\underline{\text { Min }} \quad \underline{A v g} \quad \underline{M a x} \quad \underline{\mathrm{Nb}} \quad \underline{\%} \quad \underline{\mathrm{Nb}} \quad \%$

$\begin{array}{llllllll}315 & 1 & 10.14 & 100 & 2990 & 70.75 & 2950 & 69.81\end{array}$

$\begin{array}{llllllll}314 & 1 & 10.11 & 98 & 2987 & 64.65 & 2976 & 64.42\end{array}$

304

398

$\begin{array}{lllllll}10.47 & 100 & 2985 & 72.24 & 2932 & 70.96\end{array}$

$\begin{array}{lllllll}1 & 8.36 & 82 & 3020 & 57.61 & 3009 & 57.4\end{array}$

288

376

331

404

317

313

357

298

375

290

307

308

316

260

264

405

302

293

301

311

277

272

382

$\begin{array}{lllllll}1 & 10.81 & 98 & 2933 & 69.75 & 2871 & 68.28\end{array}$

$\begin{array}{lllllll}1 & 11.78 & 104 & 2897 & 67.09 & 2845 & 65.89\end{array}$
CDS Nb Replicon Name

4226 Escherichia coli str. K-12 substr. W3110 AC_000091

4620 Escherichia coli 536 NC_008253

4132 Escherichia coli str. K-12 substr. MG1655 NC_000913

5242 Escherichia coli O157:H7 str. EC4076 NZ ABHQ

Salmonella enterica subsp. enterica serovar Typhimurium str. LT2

4423 NC_003197

5232 Escherichia coli O157:H7 str. EC4042 NZ ABHM

4266 Escherichia fergusonii ATCC 35469 NC_011740

5429 Escherichia coli O157:H7 str. EC4486 NZ ABHS

4378 Escherichia coli HS NC_009800

Salmonella enterica subsp. enterica serovar Newport str. SL254

4612 NC_011080

4732 Escherichia coli IAI39 NC 011750

Salmonella enterica subsp. enterica serovar Dublin str. CT_02021853

$\begin{array}{cccccccl}10.47 & 117 & 2943 & 65.2 & 2869 & 63.56 & 4514 & \text { NC_011205 } \\ 8.72 & 84 & 2971 & 57.11 & 2958 & 56.86 & 5202 & \text { Escherichia coli O157:H7 str. EC4206 NZ_ABHK }\end{array}$

4205 Salmonella enterica subsp. enterica serovar Enteritidis str. NC_011294

Salmonella enterica subsp. enterica serovar Virchow str. SL491

4654 NZ_ABFH

Salmonella enterica subsp. enterica serovar Heidelberg str. SL476

$\begin{array}{llllllllll}1 & 10.13 & 118 & 2931 & 63.03 & 2856 & 61.42 & 4650 & \text { NC_011083 }\end{array}$

Salmonella enterica subsp. enterica serovar Newport str. SL317

$\begin{array}{lllllllll}1 & 9.85 & 98 & 2919 & 61.8 & 2855 & 60.45 & 4723 & \text { NZ_ABEW }\end{array}$

4318 Salmonella enterica subsp. enterica serovar Typhi str. Ty2 NC_004631

$\begin{array}{lllllll}1 & 11.67 & 104 & 2899 & 65.96 & 2865 & 65.19\end{array}$

$\begin{array}{lllllll}1 & 7.91 & 79 & 2952 & 57.83 & 2897 & 56.75\end{array}$

4395

Salmonella enterica subsp. enterica serovar Typhi str. CT18 NC_003198

Escherichia coli E22 NZ_AAJV

Salmonella enterica subsp. enterica serovar Agona str. SL483

$\begin{array}{lllllllll}1 & 10.28 & 97 & 2901 & 63.59 & 2839 & 62.23 & 4562 & \text { NC_011149 }\end{array}$

Salmonella enterica subsp. enterica serovar Schwarzengrund str.

$\begin{array}{lllllllll}1 & 10.59 & 82 & 2910 & 64.67 & 2843 & 63.18 & 4500 & \text { NC_011094 }\end{array}$

Salmonella enterica subsp. enterica serovar Saintpaul str. SARA23

$\begin{array}{lllllllll}1 & 10.29 & 119 & 2901 & 65 & 2838 & 63.59 & 4463 & \text { NZ }\end{array}$

$\begin{array}{lllllll}10.17 & 99 & 2896 & 70.19 & 2909 & 70.5 & 4126\end{array}$ Escherichia coli str. K-12 substr. DH10B NC_010473

$\begin{array}{llllllll}1 & 10.96 & 97 & 2908 & 65.9 & 2831 & 64.15 & 4413\end{array}$ Salmonella enterica subsp. enterica serovar Choleraesuis str. NC_006905

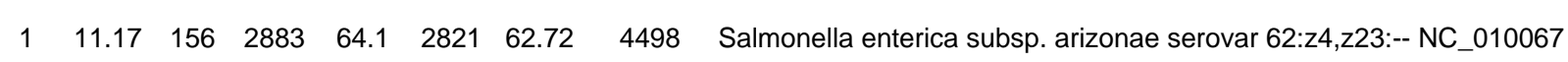

$\begin{array}{llllllll}1 & 8.28 & 62 & 2929 & 62.27 & 2888 & 61.39 & 4704\end{array}$ Escherichia coli F11 NZ_AAJU 


\begin{tabular}{|c|c|c|c|c|c|c|c|c|c|c|c|c|}
\hline $\begin{array}{l}\text { CDS in } \\
\text { Syntons }\end{array}$ & \multicolumn{2}{|c|}{ BBH } & \multirow[t]{2}{*}{$\underline{\text { Synton } \mathrm{Nb}}$} & \multicolumn{3}{|c|}{$\begin{array}{l}\text { Synton size (gene } \\
\qquad \mathrm{Nb})\end{array}$} & \multicolumn{2}{|c|}{ BBH } & \multicolumn{2}{|c|}{$\begin{array}{l}\text { CDS in } \\
\text { Syntons }\end{array}$} & \multirow[t]{2}{*}{$\underline{\text { CDS Nb }}$} & \multirow[t]{2}{*}{$\underline{\text { Replicon Name }}$} \\
\hline$\underline{\%}$ & $\underline{\mathrm{Nb}}$ & $\underline{\%}$ & & $\underline{\operatorname{Min}}$ & $\underline{\text { Avg }}$ & $\underline{\operatorname{Max}}$ & $\underline{\mathrm{Nb}}$ & $\underline{\%}$ & $\underline{\mathrm{Nb}}$ & $\underline{\%}$ & & \\
\hline 85167.13 & 2946 & 69.37 & 414 & 1 & 7.77 & 82 & 2946 & 56.82 & 2902 & 55.97 & 5185 & Escherichia coli O157:H7 str. EC4401 NZ_ABHR \\
\hline $846 \quad 67.01$ & 2900 & 68.28 & 389 & 1 & 8.1 & 72 & 2900 & 62.95 & 2842 & 61.69 & 4607 & $\begin{array}{l}\text { Escherichia coli 101-1 NZ_AAMK } \\
\text { Salmonella enterica subsp. enterica serovar Paratyphi A str. ATCC }\end{array}$ \\
\hline $46 \quad 67.01$ & 2890 & 68.05 & 255 & 1 & 11.8 & 105 & 2890 & 70.61 & 2804 & 68.51 & 4093 & NC_006511 \\
\hline $6 \quad 67.01$ & 2881 & 67.84 & 277 & 1 & 11 & 105 & 2881 & 70.65 & 2798 & 68.61 & 4078 & $\begin{array}{l}\text { Salmonella enterica subsp. enterica serovar Paratyphi A str. NC_011147 } \\
\text { Salmonella enterica subsp. enterica serovar Kentucky str. CVM29188 }\end{array}$ \\
\hline 266.92 & 2879 & 67.79 & 303 & 1 & 10.22 & 97 & 2879 & 63.25 & 2843 & 62.46 & 4552 & NZ_ABAK \\
\hline $24 \quad 66.49$ & 2898 & 68.24 & 319 & 1 & 9.66 & 99 & 2898 & 65.45 & 2851 & 64.39 & 4428 & $\begin{array}{l}\text { Escherichia coli APEC O1 NC_008563 } \\
\text { Salmonella enterica subsp. enterica serovar Hadar str. RI_05P066 }\end{array}$ \\
\hline 866.35 & 2862 & 67.39 & 325 & 1 & 9.4 & 72 & 2862 & 63.93 & 2795 & 62.43 & 4477 & NZ_ABFG \\
\hline $4 \quad 66.02$ & 2910 & 68.52 & 415 & 1 & 7.64 & 69 & 2910 & 57.37 & 2853 & 56.25 & 5072 & Escherichia coli O157:H7 str. EC4196 NZ_ABHO \\
\hline 66 & 2895 & 68.17 & 408 & 1 & 7.77 & 79 & 2895 & 58.67 & 2871 & 58.19 & 4934 & Escherichia coli E110019 NZ_AAJW \\
\hline 65.83 & 2856 & 67.25 & 325 & 1 & 9.34 & 79 & 2856 & 60.41 & 2772 & 58.63 & 4728 & Salmonella enterica subsp. enterica serovar Weltevreden str. NZ_ABFF \\
\hline 265.51 & 2815 & 66.28 & 270 & 1 & 11.04 & 95 & 2815 & 71 & 2753 & 69.43 & 3965 & NC_011274 \\
\hline 65.34 & 2836 & 66.78 & 351 & 1 & 8.61 & 77 & 2836 & 61.63 & 2760 & 59.97 & 4602 & Salmonella enterica subsp. enterica serovar 4,[5],12:i:- str. NZ_ABAO \\
\hline 65.32 & 2821 & 66.42 & 312 & 1 & 9.54 & 102 & 2821 & 66.86 & 2756 & 65.32 & 4219 & $\begin{array}{l}\text { Shigella sonnei Ss046 NC_007384 } \\
\text { Salmonella enterica subsp. enterica serovar Heidelberg str. SL486 }\end{array}$ \\
\hline 7265.27 & 2829 & 66.61 & 319 & 1 & 9.41 & 76 & 2829 & 63.27 & 2750 & 61.51 & 4471 & $\begin{array}{l}\text { NZ_ABEL } \\
\text { Salmonella enterica subsp. enterica serovar Schwarzengrund str. }\end{array}$ \\
\hline $0 \quad 65.22$ & 2824 & 66.49 & 317 & 1 & 9.5 & 79 & 2824 & 62.9 & 2755 & 61.36 & 4490 & NZ_ABEJ \\
\hline 6064.99 & 2863 & 67.41 & 389 & 1 & 7.9 & 76 & 2863 & 58.07 & 2783 & 56.45 & 4930 & Escherichia coli B171 NZ_AAJX \\
\hline 64.96 & 2867 & 67.51 & 417 & 1 & 7.35 & 68 & 2867 & 61.68 & 2781 & 59.83 & 4648 & $\begin{array}{l}\text { Escherichia coli B7A NZ_AAJT } \\
\text { Salmonella enterica subsp. enterica serovar Saintpaul str. SARA29 }\end{array}$ \\
\hline $8 \quad 64.94$ & 2799 & 65.91 & 353 & 1 & 8.51 & 95 & 2799 & 62.63 & 2734 & 61.18 & 4469 & NZ_ABAN \\
\hline 64.89 & 2868 & 67.53 & 425 & 1 & 7.38 & 64 & 2868 & 57.3 & 2813 & 56.2 & 5005 & $\begin{array}{l}\text { Escherichia coli O157:H7 str. EC4113 NZ_ABHP } \\
\text { Salmonella enterica subsp. enterica serovar Typhi str. E02-1180 }\end{array}$ \\
\hline 364.59 & 2787 & 65.62 & 374 & 1 & 7.87 & 81 & 2787 & 62.03 & 2778 & 61.83 & 4493 & NZ_CAAT \\
\hline $2727 \quad 64.21$ & 3016 & 71.01 & 482 & 1 & 6.32 & 89 & 3016 & 61.66 & 2753 & 56.29 & 4891 & Serratia proteamaculans 568 NC_009832 \\
\hline 64.12 & 2856 & 67.25 & 423 & 1 & 7.23 & 66 & 2856 & 55.74 & 2761 & 53.88 & 5124 & Escherichia coli O157:H7 str. EC4501 NZ_ABHT \\
\hline 64 & 2764 & 65.08 & 285 & 1 & 10.15 & 142 & 2764 & 67.17 & 2695 & 65.49 & 4115 & $\begin{array}{l}\text { Shigella flexneri } 5 \text { str. } 8401 \text { NC_008258 } \\
\text { Salmonella enterica subsp. enterica serovar Typhi str. E00-7866 }\end{array}$ \\
\hline 63.93 & 2814 & 66.26 & 615 & 1 & 4.86 & 32 & 2814 & 53.42 & 2798 & 53.11 & 5268 & NZ_CAAR \\
\hline $698 \quad 63.53$ & 2740 & 64.52 & 302 & 1 & 9.56 & 143 & 2740 & 65.6 & 2676 & 64.07 & 4177 & Shigella flexneri 2a str. 301 NC_004337 \\
\hline
\end{tabular}


QUERY

CDS in
Syntons

$\underline{\mathrm{Nb}} \quad \underline{\%} \quad \underline{\mathrm{Nb}} \quad \underline{\%}$

$\begin{array}{llll}2687 & 63.27 & 2713 \quad 63.88\end{array}$

$\begin{array}{llll}2680 & 63.1 & 2767 & 65.15\end{array}$ $\begin{array}{llll}2677 & 63.03 & 2721 & 64.07\end{array}$ $\begin{array}{llll}2672 & 62.91 & 2712 & 63.86\end{array}$ $\begin{array}{lllll}2671 & 62.89 & 2741 & 64.54\end{array}$ $\begin{array}{llll}2649 & 62.37 & 2685 & 63.22\end{array}$ $\begin{array}{llll}2636 & 62.07 & 2855 & 67.22\end{array}$ $\begin{array}{llll}2629 & 61.9 & 2684 & 63.2\end{array}$ $\begin{array}{llll}2562 & 60.32 & 2611 & 61.48\end{array}$ $\begin{array}{llll}2553 & 60.11 & 2795 & 65.81\end{array}$

$\begin{array}{llll}2550 & 60.04 & 2744 & 64.61\end{array}$ $\begin{array}{llll}2541 & 59.83 & 2789 & 65.67\end{array}$ $\begin{array}{llll}2498 & 58.82 & 2786 & 65.6\end{array}$ $\begin{array}{llll}2486 & 58.54 & 2676 & 63.01\end{array}$ $\begin{array}{llll}2440 & 57.45 & 2636 & 62.07\end{array}$ $\begin{array}{llll}2389 & 56.25 & 2595 & 61.1\end{array}$ $\begin{array}{lllll}2383 & 56.11 & 2576 & 60.65\end{array}$ $\begin{array}{llll}2376 & 55.95 & 2587 & 60.91\end{array}$ $\begin{array}{lllll}2361 & 55.59 & 2574 & 60.61\end{array}$

$\begin{array}{llll}2356 & 55.47 & 2777 & 65.39\end{array}$ $\begin{array}{lllll}2334 & 54.96 & 2524 & 59.43\end{array}$ $\begin{array}{llll}2332 & 54.91 & 2482 & 58.44\end{array}$ $\begin{array}{lllll}2332 & 54.91 & 2518 & 59.29\end{array}$ $\begin{array}{llll}2311 & 54.41 & 2509 & 59.08\end{array}$

$\begin{array}{llll}2289 & 53.9 & 2729 & 64.26\end{array}$ $\begin{array}{llll}2270 & 53.45 & 2481 & 58.42\end{array}$ $\begin{array}{llll}2263 & 53.28 & 2474 & 58.25\end{array}$ $\begin{array}{lllll}2261 & 53.24 & 2456 & 57.83\end{array}$ $\begin{array}{llll}2259 & 53.19 & 2462 & 57.97\end{array}$ $\begin{array}{lllll}2251 & 53 & 2467 & 58.09\end{array}$ $\begin{array}{llll}2250 & 52.98 & 2434 & 57.31\end{array}$ $\begin{array}{lllll}2250 & 52.98 & 2459 & 57.9\end{array}$ $\begin{array}{llll}2243 & 52.81 & 2443 & 57.52\end{array}$ $\begin{array}{llll}2241 & 52.77 & 2427 & 57.15\end{array}$
SYNTONS

\section{Synton size (gene BBH CDS in}

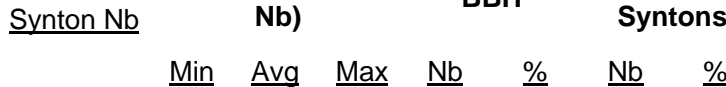

408

\subsection{9}

594

295

285

325

295

466

370

315

375

803

467

476

471

453

344

391

346

345

1031

366

331

348

337

1016

333

338

388

386

330

383

366

383

392 $\begin{array}{llllll}4.98 & 25 & 2767 & 54.89 & 2796 & 55.47\end{array}$

$\begin{array}{llllllll}1 & 9.74 & 92 & 2721 & 65.82 & 2664 & 64.44\end{array}$

$\begin{array}{lllllll}1 & 9.95 & 143 & 2712 & 66.78 & 2636 & 64.91\end{array}$

$\begin{array}{llllllll}1 & 8.94 & 94 & 2741 & 62.49 & 2642 & 60.24\end{array}$

$\begin{array}{llllllll}1 & 9.57 & 150 & 2685 & 63.24 & 2640 & 62.18\end{array}$

$\begin{array}{lllllll}1 & 6.32 & 124 & 2855 & 68.5 & 2670 & 64.06\end{array}$

$\begin{array}{llllllll}1 & 7.62 & 69 & 2684 & 60.74 & 2612 & 59.11\end{array}$

$\begin{array}{lllllll}1 & 8.63 & 71 & 2611 & 61.13 & 2551 & 59.73\end{array}$

$\begin{array}{lllllll}1 & 7.25 & 142 & 2795 & 70.26 & 2521 & 63.37\end{array}$

$\begin{array}{lllllll}1 & 3.57 & 18 & 2744 & 40.69 & 2724 & 40.39\end{array}$

$\begin{array}{llllllll}1 & 6.04 & 57 & 2789 & 71.77 & 2552 & 65.67\end{array}$

$\begin{array}{lllllll}1 & 5.76 & 89 & 2786 & 62.3 & 2518 & 56.31\end{array}$

$\begin{array}{llllllll}1 & 5.95 & 57 & 2676 & 71.38 & 2527 & 67.4\end{array}$

$\begin{array}{lllllll}1 & 6.01 & 67 & 2636 & 73.65 & 2476 & 69.18\end{array}$

$\begin{array}{lllllll}1 & 7.42 & 83 & 2595 & 61.9 & 2370 & 56.54\end{array}$

$\begin{array}{llllllll}1 & 6.72 & 83 & 2576 & 62.07 & 2370 & 57.11\end{array}$

$\begin{array}{llllllll}1 & 7.41 & 83 & 2587 & 66.32 & 2376 & 60.91\end{array}$

$\begin{array}{lllllll}1 & 7.3 & 82 & 2574 & 62.42 & 2342 & 56.79\end{array}$

$\begin{array}{lllllll}1 & 2.67 & 15 & 2777 & 37.87 & 2607 & 35.55\end{array}$

$\begin{array}{lllllll}1 & 6.82 & 60 & 2524 & 60.57 & 2364 & 56.73\end{array}$

$\begin{array}{lllllll}1 & 7.68 & 69 & 2482 & 72.42 & 2291 & 66.85\end{array}$

$\begin{array}{lllllll}1 & 7.15 & 60 & 2518 & 63.25 & 2344 & 58.88\end{array}$

$\begin{array}{lllllll}1 & 7.28 & 59 & 2509 & 65.17 & 2295 & 59.61\end{array}$

$\begin{array}{lllllll}1 & 2.61 & 14 & 2729 & 38.5 & 2500 & 35.27\end{array}$

$\begin{array}{llllllll}1 & 7.26 & 60 & 2481 & 63.76 & 2250 & 57.83\end{array}$

$\begin{array}{lllllll}1 & 7.14 & 60 & 2474 & 60.55 & 2253 & 55.14\end{array}$

$\begin{array}{lllllll}1 & 6.39 & 59 & 2456 & 58.13 & 2238 & 52.97\end{array}$

$\begin{array}{lllllll}1 & 6.42 & 59 & 2462 & 57.85 & 2238 & 52.58\end{array}$

$\begin{array}{llllllll}1 & 7.26 & 58 & 2467 & 63.5 & 2228 & 57.35\end{array}$

$\begin{array}{lllllll}1 & 6.44 & 59 & 2434 & 58.78 & 2233 & 53.92\end{array}$

$\begin{array}{lllllll}1 & 6.72 & 58 & 2459 & 63.85 & 2223 & 57.73\end{array}$

$\begin{array}{lllllll}1 & 6.42 & 58 & 2443 & 55.57 & 2229 & 50.71\end{array}$

$\begin{array}{lllllll}1 & 6.27 & 59 & 2427 & 57.95 & 2225 & 53.13\end{array}$
CDS Nb Replicon Name

Salmonella enterica subsp. enterica serovar Typhi str. E98-3139 4304 NZ_CAAZ

5041 Salmonella enterica subsp. enterica serovar Typhi str. J185 NZ_CAAW

4134 Shigella boydii Sb227 NC_007613

4061 Shigella flexneri 2a str. 2457T NC_004741

4386 Escherichia albertii TW07627 NZ_ABKX

4246 Shigella boydii CDC 3083-94 NC_010658

4168 Yersinia frederiksenii ATCC 33641 NZ_AALE

4419 Shigella dysenteriae 1012 NZ_AAMJ

4271 Shigella dysenteriae Sd197 NC_007606

3978 Yersinia enterocolitica subsp. enterocolitica 8081 NC_008800

6744 Salmonella enterica subsp. enterica serovar Typhi str. M223 NZ CAAX

3886 Yersinia intermedia ATCC 29909 NZ_AALF

4472 Pectobacterium atrosepticum SCRI1043 NC_004547

3749 Yersinia mollaretii ATCC 43969 NZ_AALD

3579 Yersinia bercovieri ATCC 43970 NZ AALC

4192 Yersinia pseudotuberculosis YPIII NC_010465

4150 Yersinia pseudotuberculosis PB1/+ NC_010634

3901 Yersinia pseudotuberculosis IP 32953 NC_006155

4124 Yersinia pseudotuberculosis IP 31758 NC_009708 Salmonella enterica subsp. enterica serovar Typhi str. E98-0664

7333 NZ CAAU

4167 Yersinia pestis Antiqua NC_008150

3427 Erwinia tasmaniensis Et1/99 NC_010694

3981 Yersinia pestis Nepal516 NC_008149

3850 Yersinia pestis Pestoides F NC 009381

Salmonella enterica subsp. enterica serovar Typhi str. E98-2068

7089 NZ CAAV

3891 Yersinia pestis biovar Microtus str. 91001 NC_005810

4086 Yersinia pestis KIM NC 004088

4225 Yersinia pestis biovar Antiqua str. E1979001 NZ_AAYV

4256 Yersinia pestis biovar Antiqua str. B42003004 NZ_AAYU

3885 Yersinia pestis CO92 NC_003143

4141 Yersinia pestis biovar Mediaevalis str. K1973002 NZ AAYT

3851 Yersinia pestis CA88-4125 NZ_ABCD

4396 Yersinia pestis biovar Orientalis str. MG05-1020 NZ AAYS

4188 Yersinia pestis biovar Antiqua str. UG05-0454 NZ_AAYR 


\section{QUERY}

CDS in

Syntons

BBH

$\underline{\mathrm{Nb}} \quad \underline{\mathrm{Nb}} \quad \underline{\%}$

$\begin{array}{llll}2227 & 52.44 & 2428 & 57.17\end{array}$

$\begin{array}{llll}2206 & 51.94 & 2396 & 56.42\end{array}$

$\begin{array}{llll}2205 & 51.92 & 2426 & 57.12\end{array}$

$\begin{array}{llll}2199 & 51.78 & 2293 & 53.99\end{array}$

\section{SYNTONS}

Synton size (gene

$\mathrm{Nb})$

Min Avg

$372-1-6.53$

$\begin{array}{llllllll}352 & 1 & 6.63 & 56 & 2396 & 62.53 & 2179 & 56.86\end{array}$

390
465

CDS in

$\begin{array}{ccccccc}1 & 6.2 & 59 & 2426 & 53.37 & 2190 & 48.17 \\ 1 & 5.26 & 47 & 2293 & 37.88 & 2777 & 45.88\end{array}$
Syntons CDS Nb Replicon Name
4254 Yersinia pestis biovar Orientalis str. F1991016 NZ_ABAT

3832 Yersinia pestis Angola NC_010159

4546 Yersinia pestis biovar Orientalis str. IP275 NZ_AAOS

6053 Sodalis glossinidius 'morsitans' chromosome SG NC_007712 